\title{
Pulse Wave Velocity in the Carotid Artery
}

Sørensen, Gertrud Laura; Jensen, Julie Brinck; Udesen, Jesper; Holfort, Iben Kraglund; Jensen, Jørgen Arendt

\section{Published in:}

Proceedings of the IEEE International Ultrasonics Symposium

Link to article, DOI:

10.1109/ULTSYM.2008.0336

Publication date:

2008

Document Version

Publisher's PDF, also known as Version of record

Link back to DTU Orbit

Citation (APA):

Sørensen, G. L., Jensen, J. B., Udesen, J., Holfort, I. K., \& Jensen, J. A. (2008). Pulse Wave Velocity in the Carotid Artery. In Proceedings of the IEEE International Ultrasonics Symposium (pp. 1386-1389). IEEE. https://doi.org/10.1109/ULTSYM.2008.0336

\section{General rights}

Copyright and moral rights for the publications made accessible in the public portal are retained by the authors and/or other copyright owners and it is a condition of accessing publications that users recognise and abide by the legal requirements associated with these rights.

- Users may download and print one copy of any publication from the public portal for the purpose of private study or research.

- You may not further distribute the material or use it for any profit-making activity or commercial gain

- You may freely distribute the URL identifying the publication in the public portal

If you believe that this document breaches copyright please contact us providing details, and we will remove access to the work immediately and investigate your claim 


\title{
Pulse Wave Velocity in the Carotid Artery
}

\author{
Gertrud Laura Sørensen ${ }^{1}$, Julie Brinck Jensen ${ }^{1}$, Jesper Udesen ${ }^{2}$, Iben Kraglund Holfort ${ }^{1}$ and \\ Jørgen Arendt Jensen ${ }^{1}$
}

(1) Center for Fast Ultrasound Imaging, Dept. of Electrical Engineering, DTU, Bldg. 349, Technical University of Denmark, DK-2800 Kgs. Lyngby, Denmark

(2) GN ReSound A/S, Lautrupbjerg 9, DK-2750 Ballerup, Denmark

\begin{abstract}
The pulse wave velocity (PWV) in the carotid artery (CA) has been estimated based on ultrasound data collected by the experimental scanner RASMUS at DTU. Data is collected from one test subject using a frame rate $(\mathrm{FR})$ of $4000 \mathrm{~Hz}$. The influence of FRs is also investigated.

The PWV is calculated from distension wave forms (DWF) estimated using cross-correlation. The obtained velocities give results in the area between $3-4 \mathrm{~m} / \mathrm{s}$, and the deviations between estimated PWV from two beats of a pulse are around $10 \%$. The results indicate that the method presented is applicable for detecting the local PWV. Additional studies with data collections from several test subjects are required to determine the accuracy of the approach. Based on a spectrum analysis it appears that there is no gain from using FRs above $1000 \mathrm{~Hz}$, but it is shown that FRs below $1000 \mathrm{~Hz}$ do not give accurate PWVs.
\end{abstract}

\section{INTRODUCTION}

Arteriosclerosis is a very common cause of death in industrialized countries. It's a disease where deposits of lipids makes a plaque in the inner layer of the arterial wall. Advanced arteriosclerosis can occlude the arteries and involve changes of the vessel wall that reduce its elasticity and flexibility. The disease may not give symptoms before $2 / 3$ of the vessel is occluded. A measure of the degree of arteriosclerosis is therefore valuable, since the disease thereby can be discovered at an earlier stage before it has fatal consequences.

When the heart contracts a pressure pulse wave will propagate through the arteries. The pulse wave velocity (PWV) can be used as a measure of the degree of arteriosclerosis, due to the relation between PWV and the stiffening of the arteries. This relation is described by the Moens-Korteweg equation [1]:

$$
\mathrm{PWV}=\sqrt{\frac{E \cdot h}{\rho \cdot d}},
$$

where $E$ is the wall elastic modulus, $h$ the wall thickness, $\rho$ the blood density, and $d$ the lumen diameter. When a vessel is occluded the lumen radius will be reduced and the wall elastic modulus will increase. As a result the PWV will also increase. Therefore it is desirable to find methods for estimating the PWV locally. The PWV is $3-4 \mathrm{~m} / \mathrm{s}$ in the aorta and even faster in smaller arteries, so a fast data acquisition is needed to properly estimate the velocity.

Previous studies have proved the connection between the stiffening of the arteries and the PWV. As early as 1878 Moens [2] derived the mathematical equation for the PWV described in 1. In 1954 Edler and Hertz [2] used ultrasound to detect the movement of tissue structures by one-dimensional M-mode recordings. The real technical breakthrough, however, came with the development of the Time-Distance-recorder [3], a device for measuring, as a function of time, the relative movements of two echoes visualized by a two-dimensional, real-time ultrasound scanner.

In 1991 Benthin et al. [2] recorded ultrasound measurements at two sites along an artery to detect the diameter change and produce a distension wave form (DWF). By means of cross-correlation of two DWFs, the PWV was estimated. They concluded that in order to reduce the influence of reflections from arterial bifurcations, only the foot of the DWF should be used.

In 2003 Rabben et al. [5] investigated an area-flow method for estimating local PWV from ultrasound measurements. With the area-flow method, the PWV is estimated as the ratio between change in flow and change in cross-sectional area. The ultrasound recordings were performed on the CA of 21 human subjects. They estimated PWVs in the area between $4-9 \mathrm{~m} / \mathrm{s}$ and found that the velocity is increasing with age. However, improvements in the basic measurements seem necessary to increase the precision of the method.

In 2006 Hermeling et al. [1] investigated the precision of an ultrasound method to measure the PWV. A phantom scaled according to realistic in vivo conditions was used. Three approaches were used to identify the systolic foot of the DWF, and the PWV was estimated. The method still needs additional investigation to be applicable to in vivo measurements.

In 2007 Williams et al. [4] used two methods to measure the PWV in mice. One of the methods was a regional transit-time method, where the time difference between the rise of a pulse wave at two sites was measured, while the distance between the sites was known. The other method was a local flowarea method, where the cross sectional area and the volume flow through a vessel were measured by means of color flow imaging. The PWV was estimated as the relationship between the change in the flow volume and the cross sectional area. They concluded that the two methods were appropriate to give an understanding of the arterial properties among mice, but they did not conclude on whether the method can be transferred to humans.

Common to the previous studies that measured the PWV by means of ultrasound data, is that they have used conventional ultrasound, which uses a FR of 20-50 Hz. The PWV in smaller arteries than the aorta is expected not to exceed $10 \mathrm{~m} / \mathrm{s}$. If 
the distance between two measuring sites is $1 \mathrm{~cm}$, the time between the rise of the pulse wave at the first site to the next site can be calculated to:

$$
t=\frac{0.01 \mathrm{~m}}{10 \mathrm{~m} / \mathrm{s}}=0.001 \mathrm{~s} .
$$

The highest frequency of the received signal should thus be $1000 \mathrm{~Hz}$, and the FR must at least be the double of this.

This paper investigates whether it is possible to find a local measure of the PWV using plane wave ultrasound (PWU) which uses a FR of $4 \mathrm{kHz}$. Furthermore the influence of the FR is investigated.

This work is a preliminary study. Data is collected from the carotid artery (CA) of one test subject using the experimental scanner RASMUS at DTU [8]. The PWV is calculated by measuring the time difference between two velocity profiles while knowing the distance between the measuring sites. The results are validated by comparing with results from previous studies and by comparing velocities from two different heartbeats.

\section{METHOD}

In PWU all transducer elements are excited at the same time thereby creating a pressure wave with a nearly plane wave front. The acquisition time for each frame is thereby lowered significantly, but the contrast and focusing of the image is compromised compared to conventional imaging.

The transmitted pulse consists of a 13 bit Barker code on each transducer element. The code is used to increase SNR. The image is focused using traditional delay-and-sum receive beamforming. Using the RASMUS experimental ultrasound scanner, data from the CA of a 36 year old healthy male is obtained. Data is acquired during a period of 2.2 seconds using a $5.5 \mathrm{MHz}$ linear array transducer, which yields data showing two heartbeats. The transducer set-up is shown in table I [6].

\begin{tabular}{lc}
\hline Parameter & Value \\
\hline Transducer & Linear array \\
Number of elements & 128 \\
Pitch & $0,208 \mathrm{~mm}$ \\
Pitch & $0,75 \cdot \lambda$ \\
Kerf & $0,035 \mathrm{~mm}$ \\
Height & $4,5 \mathrm{~mm}$ \\
Center frequency, $f_{0}$ & $5,5 \mathrm{MHz}$ \\
Bandwidth $(2-$ way, $-6 \mathrm{~dB})$ & $60 \%$ af $f_{0}$ \\
Elevation focus & $25 \mathrm{~mm}$ \\
Sampling frequency & $40 \mathrm{MHz}$ \\
Apodization in transmit & Tukey \\
Apodization in receive & None \\
\hline
\end{tabular}

TABLE I

TRANSDUCER SET-UP. $\lambda$ IS THE WAVELENGTH AT THE CENTER FRECUENCY.

The difference between the relative movement of the upper and the lower arterial wall gives the DWF. By cross-correlating adjacent ultrasound images, the relative movement of the arterial wall is estimated.

Two ways of calculating the DWF have been used to investigate how the influence of noise in the signals effects the results. The 1'st method cross-correlates a signal sequence from the $i$ 'th transmission with a signal sequence from the $(i+$ 1)'th transmission. In the 2 'nd method a signal sequence from the $i$ 'th transmission is cross-correlated with a signal sequence from the $(i+4)$ 'th transmission. This cross-correlation function is given by:

$$
R_{i, i+K}(\kappa)=\int_{-\infty}^{\infty} s_{i}(z) s_{i+K}(z-\kappa) d z
$$

where $\kappa$ is the spatial displacement, $s$ is the signal sequence, $i$ the transmission and $K$ is either 1 or 4 and indicates the gap between the cross-correlated transmissions. When $K=4$ it does not corresponds to a FR which is 4 times less the one which is used, because all the data is still used, but the detected relative movement of the vessel wall is bigger, which gives rise to a DWF less influenced by noise.

The PWV is estimated as the distance, $\Delta x$ between two measuring sites divided by the time difference, $\Delta t$, between the arise of a pulse wave at these two sites:

$$
P W V=\frac{\Delta x}{\Delta t} .
$$

The time difference, $\Delta t$, is found from the delay between two DWFs at two different spatial positions along the artery. The delay is obtained from the sum-of-squared difference estimator [7]:

$$
S_{x y}(l)=\sum_{n=1}^{N}(x(n)-y(n-l))^{2}, \quad l=0, \pm 1, \pm 2, \ldots .
$$

where $l$ is the spatial displacement and $y$ and $x$ are the two DWFs.

To reduce the influence of reflections from arterial bifurcations, only a part of the DWF is used in the calculation. These parts are the systolic part of one of the DWFs and the foot of the systolic part of the other DWF. In previous studies it is shown that exactly these parts of the DWFs will reduce the influence from reflections [2].

To obtain the most correct DWF, a spatial averaging has been performed, by calculating the average DWF of 3 DWFs from adjacent signal sequences:

$$
D W F=\frac{D W F_{x-1}+D W F_{x}+D W F_{x+1}}{3} .
$$

Furthermore the mean of this new DWF is found and this mean value is subtracted from the very same DWF:

$$
D W F=D W F-\frac{1}{N} \sum_{n=1}^{N} D W F(n),
$$

where $z$ indicates the elements in the DWF and $N$ is the number of elements in the DWF.

The last modification is to avoid difference in the amplitude values at the beginning of the systolic foot of two DWFs used to calculate the PWV. This modification corresponds to removing the $0 \mathrm{~Hz}$ component of the spectrum of the DWF. To compare the different methods used to estimate the PWVs, the relative deviation between two estimated PWVs of two different heartbeats from the same pair of estimated DWFs, is calculated. In this calculation the PWV from the first heartbeat is subtracted from the PWV from the second 
heartbeat, and the subtraction is divided by the PWV from the first heartbeat. This calculation is performed for all pairs of DWFs and the mean of each pair is calculated to evaluate which method is the most accurate.

To investigate whether the high FR improves the estimation of the PWV, the measured signals have been resampled to obtain lower FRs. This is done by using only a subset of the image frames, calculate new DWFs and estimate the PWV. From this investigation a graph is produced with the estimated PWV's as a function of FR.

\section{RESUlTS}

When cross-correlating the signal sequence from the $i$ 'th transmission with a signal sequence from the $(i+1)$ 'th transmission and signal sequence from the $i$ 'th transmission with the signal sequence from the $(i+4)$ 'th transmission different DWFs are obtained. This is illustrated in Figs. 1 and 2. It can be seen that the DWF calculated from the second cross-correlation method is more smooth, which is consistent with the fact that the detected relative movement of the vessel wall in this method is bigger.

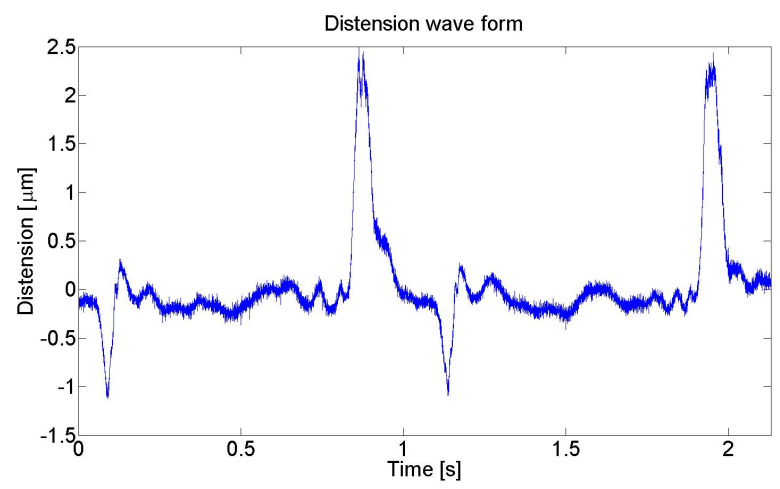

Fig. 1. DWF calculated by cross-correlating the signal sequence from the $i$ 'th transmission with a signal sequence from the $(i+1)$ 'th transmission

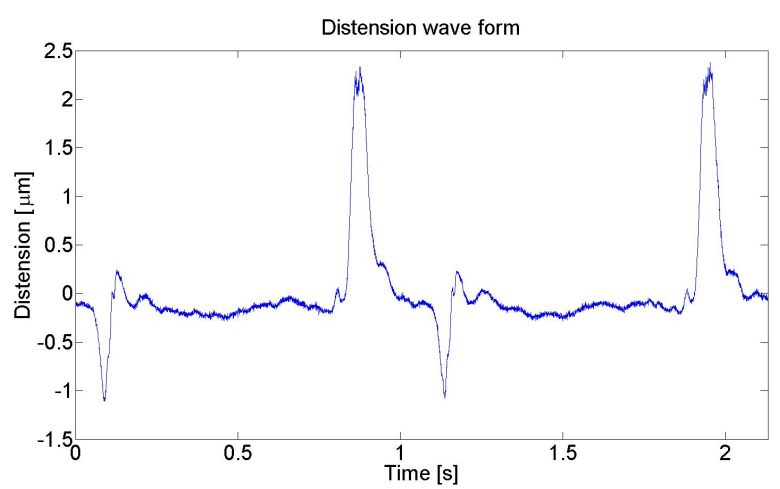

Fig. 2. DWF calculated by cross-correlating the signal sequence from the $i$ 'th transmission with a signal sequence from the $(i+4)$ 'th transmission

The DWFs that appear when modifications has been performed according to the methods mentioned in section II also look slightly different than the ones shown in Figs.
1 and 2. Therefore different values of PWVs are obtained, when the velocities are calculated from DWFs with different modifications and different cross-correlation methods. The values of the PWVs are shown in Table II.

\begin{tabular}{|c|c|c|c|c|}
\hline \multirow[t]{2}{*}{ Method } & \multicolumn{2}{|c|}{$\begin{array}{c}\text { 1'st cross-correlation } \\
\text { method }\end{array}$} & \multicolumn{2}{|c|}{$\begin{array}{c}\text { 2'nd cross-correlation } \\
\text { method }\end{array}$} \\
\hline & $\begin{array}{l}\text { 1'st heart- } \\
\text { beat }[\mathrm{m} / \mathrm{s}]\end{array}$ & $\begin{array}{l}\text { 2'nd heart- } \\
\text { beat }[\mathrm{m} / \mathrm{s}]\end{array}$ & $\begin{array}{l}\text { 1'st heart- } \\
\text { beat }[\mathrm{m} / \mathrm{s}]\end{array}$ & $\begin{array}{l}\text { 2'nd heart- } \\
\text { beat }[\mathrm{m} / \mathrm{s}]\end{array}$ \\
\hline $\begin{array}{c}\text { Without } \\
\text { modification }\end{array}$ & 2.50 & 2.18 & 3.57 & 3.36 \\
\hline $\begin{array}{c}\text { Spatial } \\
\text { averaging }\end{array}$ & 2.66 & 2.49 & 3.57 & 3.33 \\
\hline $\begin{array}{c}\text { Spatial } \\
\text { averaging, } \\
\text { subtraction } \\
\text { of } \mu\end{array}$ & 3.27 & 3.07 & 3.77 & 3.54 \\
\hline
\end{tabular}

TABLE II

PWVS CALCULATED FROM DWFS WITH DIFFERENT MODIFICATIONS. FOR EACH METHOD THE PWV HAS BEEN CALCULATED FROM 10 DIFFERENT

PAIRS OF DWFS. THIS TABLE SHOWS THE MEAN VALUE OF THESE CALCULATIONS. IN THE TABLE $\mu$ REPRESENTS THE MEAN VALUE OF THE SPATIAL AVERAGED DWFS.

The results of the PWVs given in Table II are low compared the velocities obtained in previous studies around $4-9 \mathrm{~m} / \mathrm{s}$ [5]. The method that gives velocities closest to the ones given in the literature is when using the second cross-correlation method, spatially averaging the DWFs and then subtracting the mean value of the spatial averaged DWF.

In Table III the relative deviations of the PWVs of two successive heartbeats are shown. PWVs in the table are estimated by using DWFs which have been cross-correlated and modified in different ways. Hereby it is possible to see which method yields the smallest deviation.

\begin{tabular}{|c|c|c|}
\hline Method & $\begin{array}{c}\text { 1'st cross-correlation } \\
\text { method }\end{array}$ & $\begin{array}{c}\text { 2'nd cross-correlation } \\
\text { method }\end{array}$ \\
\cline { 2 - 3 } & Deviation in \% & Deviation in \% \\
\hline Without modification & 34.9 & 13.6 \\
\hline Spatial averaging & 33.2 & 11.4 \\
\hline $\begin{array}{c}\text { Spatial averaging, } \\
\text { subtraction of } \mu\end{array}$ & 28.9 & 9.65 \\
\hline
\end{tabular}

TABLE III

MEAN VALUE of THE RELATIVE DEVIaTion BETWEen THE PWV CALCULATED FROM TWO HEARTBEATS. IN THE TABLE $\mu$ REPRESENTS THE MEAN VALUE OF THE SPATIAL AVERAGED DWFS.

From (1) it is known that $E, h, \rho$ and $d$ determines the PWV. These variables remain the same for two successive heartbeats measured at the same site in the artery. Therefore the most correct PWVs must be those calculated from the method that gives the smallest deviation between the two heartbeats. From Table III it can be seen that the method that gives the smallest deviation is when the DWFs are spatially averaged and the mean value of the DWF that hereby is obtained is subtracted from the very same DWF. Furthermore the second cross-correlation method where a signal sequence from $i$ 'th transmission has been cross-correlated with the signal sequence from the $(i+4)$ 'th transmission gives 
significantly smaller deviations than the first cross-correlation method. It should be noted that the method giving the smallest deviation is also the method giving the values of PWVs closest to the ones from the literature (as shown in table II). Therefore it seems that this method produces the most correct estimates of the PWV.

In Fig. 3 a graph displaying the estimated PWVs as a function of FR is shown. When investigating the graph it is clear that when the FR becomes lower than $1 \mathrm{kHz}$ the precision of the estimated velocities drop. Thus it would not be possible to measure the PWV with conventional ultrasound. It appears, however, that the estimated PWVs does not change significantly from $1 \mathrm{kHz}$ to $4 \mathrm{kHz}$. Therefore it seems that nothing is gained by using a FR above $1 \mathrm{kHz}$.

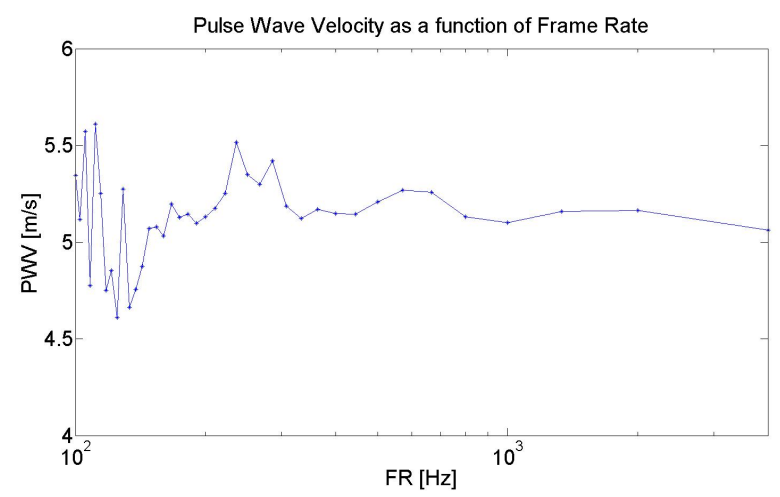

Fig. 3. PWV as a function of FR. The graph is calculated from a set of DWFs which have been spatial averaged where the mean has also been subtracted. Furthermore the cross-correlation method where a signal sequence from $i$ 'th transmission has been cross-correlated with the signal sequence from the $(i+4)$ 'th transmission has been used. A logarithmic (base 10) scale is used for the $\mathrm{X}$-axis.

\section{CONCLUSIONS}

It has been investigated whether it is possible to find local measures of the PWV in the CA using PWU. The PWU uses a FR of $4 \mathrm{kHz}$ and therefore it has also been examined whether this high FR gives rise to more correct estimates of the PWV. It is possible to find the PWV in the Carotid Artery. It has been investigated how the cross-correlation of signal sequences should be done in order to obtain the most correct PWVs. This investigation has shown that the most correct PWVs are obtained when DWFs have been calculated by crosscorrelating a signal sequence from the $i$ 'th transmission with a signal sequence from the $(i+4)$ 'th transmission instead of the signal sequence from the $i$ 'th transmission with a signal sequence from the $(i+1)$ 'th transmission. This is consistent with the fact that the detected relative movement of the vessel wall is bigger when cross-correlating with the $(i+4)$ 'th transmission, which gives rise to DWFs less influenced by noise. This paper has, however, not examined if even better estimates of the PWV could be achieved by using a value of $K$ in (3) higher than 4 . Therefore it might be an idea to examine this in future studies.

When calculating the PWV it has furthermore been found that certain modifications of the DWFs gives even better results. These modifications includes calculating spatial averaged DWFs, finding the mean-value of the obtained DWF and subtracting it from the DWF. The PWVs that has been calculated from this procedure are in the interval of $3-4$ $\mathrm{m} / \mathrm{s}$. These estimates are low compared to the ones given in previous studies and also compared fact that the PWV is $3-4$ $\mathrm{m} / \mathrm{s}$ in the aorta and should be higher in smaller arteries like the CA. An error in the calculations of PWV in this paper might be caused by reflections of the blood in the artery from arterial bifurcations or plaque on the inside of the vessel. Even though it has been tried to avoid the reflections by using only the systolic part of one of the DWFs and the foot of the systolic part of the other DWF when calculating the PWV, it can not be concluded that reflections of the blood have not contributed to an error in the calculations.

When investigating the influence of the value of the FR it has been shown that it is not suitable to use a FR below 1 $\mathrm{kHz}$. The PWV calculated from a FR under $1 \mathrm{kHz}$ will not be accurate. Furthermore it appears that no additional information is obtained by using a FR of $4 \mathrm{kHz}$ instead of $1 \mathrm{kHz}$, since the estimated PWV does not change significantly in this interval. Finally it should be mentioned that this study is limited, since data has only been collected from one test subject. To make sure that the procedure used in this paper is valid, it has to be tested on more test subjects.

\section{REFERENCES}

[1] Hermeling, E., Reesink, K.D., Reneman, R.S., and Hoeks, A.P.G., Measurement of local pulse wave velocity: Effects of signal processing on precision, Ultrasound Med. Biol., Vol. 33, no. 5, pp. 774-781, 2007.

[2] Benthin, M., Dahl, P., Ruzicka, R., and Lindström, K., Calculation of pulse-wave velocity using cross correlation - effects og reflexes in the arterial tree, Ultrasound Med. Biol., Vol. 17, No. 5, pp. 461-469, 1991.

[3] Lindström, K., Marsal, K., Gennser, G., Bengtsson, L., Benthin, M., and Dahl, P., Device for measurement for of fetal breathing movements-I: The TD-recorder. A new system for recording the distance between two echogenerating structures as a function of time, Ultrasound Med. Biol., No. 3, pp. 143-151, 1977.

[4] Williams, R., Needles, A., Cherin, E., Zhou, Y., Henkelman, R.M., Adamson, S.L., and Foster, F.S., Noninvasive ultrasonic measurement of regional and local pulse-wave velocity in mice, Ultrasound Med. Biol., Vol. 33, No. 9, pp. 1368-1375, 2007.

[5] Rabben, S.I., Stergiopulos, N., Hellevik, L.R., Smiseth, O.A., Slrdahl, S., Urheim, S., and Angelsen, B., An ultrasound-based method for determining pulse wave velocity in superficial arteries, Journal of Biomechanics, Vol. 37 , No. 10 , pp. 1615 - 1622, 2004.

[6] Udesen, J., Gran, F., Hansen, K.L., Jensen, J.A., Thomsen, C., and Nielsen, M.B., High frame-rate blood vector velocity imaging using plane waves: Simulations and preliminary experiments, Accepted for publication IEEE Trans. Ultrason., Ferroelec. and Freq. Contr., 2008.

[7] Viola, F., and Walker, W.F., A comparison of the performance of time-delay estimators in medical ultrasound, IEEE Trans. Ultrason., Ferroelec. and Freq. Contr., Vol. 50, No. 4, pp. 392-401, 2003.

[8] Jensen, J.A., Holm, O., Jensen, L.J., Bendsen, H., Nikolov, S.I., Tomov, B.G., Munk, P., Hansen, M., Salomonsen, K., Hansen, J., Gormsen, K., Pedersen, H.M., and Gammelmark, K.L, Ultrasound Research Scanner for Real-time Synthetic Aperture Data Acquisition, IEEE Trans. Ultrason., Ferroelec. and Freq. Contr., Vol. 52, No. 5, pp. 881-891, 2005. 